\title{
Phase 1b Study of Ramucirumab in Combination With Erlotinib or Osimertinib for Untreated EGFR- mutated Non-small Cell Lung Cancer Patients With Asymptomatic Brain Metastases
}

Hiroyasu Kaneda ( $\sim$ kaneda.hiroyasu@med.osaka-cu.ac.jp)

Graduate School of Meicine, Osaka City University https://orcid.org/0000-0002-7696-2391

\section{Kenji Sawa}

Osaka City University: Osaka Shiritsu Daigaku

\section{Haruko Daga}

Osaka City General Hospital: Osaka Shiritsu Sogo Iryo Center

\section{Asuka Okada}

Osaka City General Hospital: Osaka Shiritsu Sogo Iryo Center

\section{Yuki Nakatani}

Osaka City General Hospital: Osaka Shiritsu Sogo Iryo Center

\section{Shinji Atagi}

Kinki Chuo Chest Medical Center: Kokuritsu Byoin Kiko Kinki Chuo Kokyuki Center

\section{Kyoichi Okishio}

Kinki Chuo Chest Medical Center: Kokuritsu Byoin Kiko Kinki Chuo Kokyuki Center

\section{Yoko Tani}

Osaka City University: Osaka Shiritsu Daigaku

\section{Yoshiya Matsumoto}

Osaka City University: Osaka Shiritsu Daigaku

\section{Koichi Ogawa}

Osaka City University: Osaka Shiritsu Daigaku

\section{Kenji Nakahama}

Osaka City University: Osaka Shiritsu Daigaku

Motohiro Izumi

Osaka City University: Osaka Shiritsu Daigaku

\section{Shigeki Mitsuoka}

Osaka City University: Osaka Shiritsu Daigaku

\section{Tomoya Kawaguchi}

Osaka City University: Osaka Shiritsu Daigaku 


\section{Research Article}

Keywords: EGFR mutation, ramucirumab, brain metastasis, non-small cell lung cancer, antiangiogenic agent, tyrosine kinase inhibitor

Posted Date: May 27th, 2021

DOl: https://doi.org/10.21203/rs.3.rs-533997/v1

License: (c) (i) This work is licensed under a Creative Commons Attribution 4.0 International License.

Read Full License 


\section{Abstract}

Objectives: The study was designed to investigate the safety and tolerability of ramucirumab administered in combination with erlotinib or osimertinib for patients with untreated EGFR-mutated nonsmall cell lung cancer (NSCLC) and asymptomatic brain metastases, a patient subgroup in which these regimens have remained untested.

Materials and methods: This multicenter phase 1b study (RELAY-Brain) consisted of two cohorts with three patients each. The two cohorts were assessed independently of one another and were not compared. Patients whose asymptomatic brain metastases had or had not undergone prior local therapy received ramucirumab $(10 \mathrm{mg} / \mathrm{kg}$ ) every 2 weeks plus either erlotinib (150 mg/day) or osimertinib (80 $\mathrm{mg} /$ day) until disease progression or intolerable toxicity. The primary objective of the study was to assess dose-limiting toxicity (DLT), defined as central nervous system (CNS) hemorrhage of grade ${ }^{3} 2$. Secondary end points included safety profile, objective response (systemic and intracranial), and disease control rate.

Results: Six patients were enrolled in the study. Neither DLT nor serious or unexpected adverse events were observed. At the time of this analysis, discontinuation of the study drugs had also not occurred. One treatment-related adverse event of grade ${ }^{3} 3$ (hypertension of grade 3 ) was apparent. Common adverse events were generally manageable. The median number of ramucirumab administrations was 18.5 (range, 13 to 31), and there were no detected episodes of CNS hemorrhage. Four patients experienced at least one dose delay for ramucirumab, but there were no dose reductions or omissions for this drug. Five of the six patients showed an objective systemic response. Although only one patient had a measurable CNS lesion at baseline, a confirmed intracranial partial response was observed.

Conclusion: Ramucirumab in combination with erlotinib or osimertinib showed promising safety and efficacy for EGFR-mutated NSCLC with brain metastases.

Clinical trial registration: Japan Registry of Clinical Trials (jRCTs2051190027)

\section{Introduction}

Mutant forms of the epidermal growth factor receptor (EGFR) are therapeutic targets that are present in $20 \%$ to $40 \%$ of individuals with advanced non-small cell lung cancer (NSCLC) [1, 2]. EGFR tyrosine kinase inhibitors (TKIs) have thus been found to substantially prolong progression-free survival (PFS) compared with chemotherapy as well as to improve overall survival and quality of life in such individuals [3-5]. However, most of these patients eventually develop disease progression during treatment with EGFR-TKIs, in most cases as a result of the acquisition of a secondary T790M mutation in exon 20 of EGFR [6, 7]. Osimertinib is an irreversible EGFR-TKI that selectively inhibits forms of EGFR with both a TKI-sensitizing mutation (exon-19 deletion or an L858R point mutation in exon 21) and the T790M resistance mutation of EGFR. The FLAURA study revealed superior efficacy for osimertinib compared with the first-generation EGFR-TKIs gefitinib or erlotinib as a first-line treatment for advanced NSCLC [8]. In addition, osimertinib 
has consistently shown central nervous system (CNS) efficacy in several studies performed with EGFR mutation-positive NSCLC patients $[9,10]$. Despite these beneficial effects of osimertinib, however, disease progression during treatment with this drug is universal. The establishment of novel combination therapies to achieve a more durable efficacy is thus urgently needed.

Strategies to overcome EGFR-TKI resistance, such as the combination of EGFR-TKIs with antiangiogenic or cytotoxic agents, have been developed. Several prospective studies have shown that the antiangiogenic agent bevacizumab enhances the efficacy of first-generation EGFR-TKIs in patients with EGFR-mutated NSCLC [11-14]. Ramucirumab is a monoclonal antibody that binds to the extracellular domain of vascular endothelial growth factor (VEGF) receptor 2 with high specificity and affinity. Phase 3 studies have shown that it provides clinical benefit either in combination with chemotherapy for gastric cancer, colorectal cancer, and NSCLC [15-17] or as monotherapy for gastric cancer and hepatocellular carcinoma $[18,19]$. The RELAY phase 3 trial recently showed found that ramucirumab plus erlotinib significantly prolonged PFS compared with placebo plus erlotinib in patients with untreated EGFRmutated NSCLC [20]. Patients with brain metastases were excluded from this trial, however, because of concerns about potential intracranial hemorrhage. The safety of bevacizumab with regard to the induction of CNS hemorrhage in NSCLC patients with treated or untreated brain metastases has been evaluated in several trials [21-23]. The cumulative evidence suggests that the risk of such hemorrhage associated with bevacizumab treatment is low. However, the safety of ramucirumab in combination with EGFR-TKIs for EGFR-mutated NSCLC patients with brain metastases has remained unknown. We therefore performed a phase $1 \mathrm{~b}$ study to assess the safety and tolerability of ramucirumab administered in combination with erlotinib or osimertinib in patients with untreated EGFR mutation-positive NSCLC and asymptomatic brain metastases.

\section{Materials And Methods}

\subsection{Patient eligibility}

The study enrolled individuals aged 20 years or older with previously untreated EGFR mutation (exon-19 deletion or L858R) - positive stage IV NSCLC and with brain metastasis documented by magnetic resonance imaging (MRI). With regard to brain metastases, if the lesion was asymptomatic, untreated, and either had a major diameter of $<20 \mathrm{~mm}$ or was nonmeasurable according to the Response Evaluation Criteria in Solid Tumors (RECIST) version 1.1, the patient was allowed to enter the study. Patients with initially symptomatic or larger brain metastases (major diameter of ${ }^{3} 20 \mathrm{~mm}$ ) were considered eligible for the study if the lesion had received adequate prior treatment. All patients were required to be neurologically stable, and those with brain metastases with a major diameter of ${ }^{3} 40 \mathrm{~mm}$ were excluded. Eligible patients were also required to have an Eastern Cooperative Oncology Group performance status of 0 or 1 , at least one measurable lesion according to RECIST, and adequate organ function (as evaluated on the basis of hematologic, coagulation, heart, hepatic, and renal parameters). Exclusion criteria included a detected T790M mutation of EGFR, clinically relevant interstitial lung disease, uncontrolled hypertension, third-space fluid requiring frequent drainage, and leptomeningeal carcinomatosis. 
Administration of corticosteroids at a dose of $>10 \mathrm{mg} /$ day for the prevention of cerebral edema related to prior treatment was not allowed. The study was approved by the institutional review board of each participating institution and was conducted in compliance with the ethical principles of the Declaration of Helsinki, the International Council for Harmonisation Good Clinical Practice Guideline, and applicable local regulations. All patients provided written informed consent. The study was registered with the Japan Registry of Clinical Trials (jRCTs2051190027).

\subsection{Study design and treatment}

The study was designed as a noncomparative, nonrandomized, multicenter phase $1 \mathrm{~b}$ trial to assess the safety and tolerability of ramucirumab in patients with previously untreated EGFR mutation-positive NSCLC and with asymptomatic brain metastases. The study was to include two cohorts that were to be assessed independently and not compared. The target enrollment was a total of six patients. Patients received either ramucirumab plus erlotinib or ramucirumab plus osimertinib. Ramucirumab was administered intravenously at a dose of $10 \mathrm{mg} / \mathrm{kg}$ over a period of $1 \mathrm{~h}$ on day 1 of each cycle (every 2 weeks). Patients in cohorts 1 or 2 also received erlotinib at $150 \mathrm{mg}$ daily or osimertinib at $80 \mathrm{mg}$ daily, respectively. In both cohorts, patients continued the study treatment until disease progression, the development of unacceptable toxicity, noncompliance, withdrawal of consent, or termination by the investigator. Dose adjustment and treatment delays due to management of adverse events (AEs) were permitted. Ramucirumab could be delayed for up to 42 days as a result of toxicity (proteinuria or hypertension). Three steps of dose reduction for ramucirumab were allowed (to 8,6 , and $5 \mathrm{mg} / \mathrm{kg}$ ). Ramucirumab treatment was discontinued if patients developed severe AEs related to the drug (ie, grade 4 events, infusion reaction, thromboembolism, gastrointestinal bleeding or perforation, or congestive heart failure). Dose adjustment for EGFR-TKIs included a reduction of erlotinib dose in 50-mg decrements to a minimum of $50 \mathrm{mg}$ daily, and a reduction of osimertinib dose to $40 \mathrm{mg}$ once daily. Patients who discontinued ramucirumab or EGFR-TKI treatment as a result of toxicity were allowed to continue singleagent treatment with EGFR-TKIs or ramucirumab, respectively, until disease progression or unacceptable toxicity.

\subsection{Study end points and assessments}

The primary objective of the study was to investigate the safety and tolerability of ramucirumab when administered in combination with erlotinib or osimertinib-in particular, with regard to evaluation of doselimiting toxicity (DLT)-in patients with previously untreated EGFR mutation-positive NSCLC and with asymptomatic brain metastases. This patient subgroup was not examined in the RELAY study [20]. DLT was defined as CNS hemorrhage of grade $\geq 2$ (a clinically significant intracranial hemorrhage), where grade 2 is defined as CNS hemorrhage for which medical intervention is indicated. Secondary end points included safety profile and preliminary efficacy of the study treatment based on investigator-assessed objective response rate (ORR), disease control rate (DCR), and intracranial response rate. Disease assessment was performed within 28 days before initiation of study treatment as baseline, every 6 weeks after the first study dose up to 24 weeks, and then every 12 weeks until disease progression or study 
discontinuation. Tumor response was assessed by the investigator according to RECIST version 1.1. Brain tumor imaging by gadolinium-enhanced MRI was mandated for all patients. The imaging method adopted at baseline was used consistently for tumor assessment. All AEs and other symptoms were graded according to the National Cancer Institute Common Terminology Criteria for Adverse Events version 4.0.

\subsection{Statistical analysis}

The target enrollment was six patients. The primary end point was assessment of DLT in evaluable patients according to a conventional $3+3$ design. DLT was assessed during the first two cycles (4 weeks) of treatment. DLT-evaluable patients were considered to be individuals who either completed two cycles of treatment or discontinued the study treatment or study participation before completing two cycles as a result of DLT. All patients who enrolled in the study and received at least one dose of study treatment were assessed for safety. Safety was evaluated on the basis of reported AEs, clinical laboratory assessments, vital signs, and physical examinations. AEs other than CNS hemorrhage were not reassessed as DLT because the safety of ramucirumab plus erlotinib had been thoroughly assessed in part A (phase 1b portion) of the RELAY study [20]. In addition, efficacy analysis included all patients in the safety population with the exception of those found to be not eligible or those for whom efficacy data were not available. The point estimate of ORR-the proportion of patients in the efficacy population who achieved a best overall response of a partial response (PR) or a complete response (CR)-was calculated as defined by RECIST version 1.1. DCR-the proportion of patients in the efficacy population who achieved a best overall response of a PR, a CR, or stable disease (SD)-and intracranial response rate were also analyzed in the same way as ORR. Preplanned interim analysis was to be performed 6 months after the start of administration of study treatment.

\section{Results}

\subsection{Patient disposition and characteristics}

The data cutoff date was 30 November 2020. Between 4 September 2019 and 5 March 2020, a total of six patients, with three in each cohort, was enrolled in the study. All six patients received at least two cycles of study treatment without discontinuation, and they were eligible for assessment of safety including DLT as well as of efficacy. At the time of this analysis, discontinuation of the study drugs had also not occurred. The median follow-up time was 9.4 months (range, 8.2 to 14.3 months). Clinical characteristics at baseline are summarized in Table 1. The median age was 63.5 years, and most patients were male, never-smokers, and had stage IV disease at diagnosis as well as an L858R mutation in exon 21 of EGFR. Four patients had undergone surgery or radiotherapy for brain metastasis before study enrollment. All patients had adenocarcinoma.

\subsection{Assessment of DLT and safety}


An overview of safety findings including DLT assessment is provided in Table 2. CNS hemorrhage was not detected during the DLT assessment period or as of the data cutoff. All patients who received study treatment experienced one or more treatment-emergent AEs (TEAEs). TEAEs of grade 3 occurred in two patients, hypertension and anaphylaxis in one each. Only the case of grade 3 hypertension was related to ramucirumab treatment. No serious AEs occurred. Discontinuation of study treatment as a result of treatment-related AEs (TRAEs) did not occur. The most common ( ${ }^{3} 50 \%$ of patients) TEAEs of any grade were rash, dry skin, stomatitis, diarrhea, paronychia, alanine aminotransferase (ALT) increased, epistaxis, and edema (Table 3). One or more known AEs of special interest for ramucirumab were observed in four patients; the only such event of grade 33 was hypertension, which was manageable, and the other such events included bleeding or hemorrhage, liver failure or injury, and proteinuria were mild. There was no occurrence of interstitial lung disease. In addition, no new safety signals were observed.

\subsection{Treatment exposure}

At the time of this interim analysis, the median number of ramucirumab administrations was 18.5 (range, 13 to 31). With regard to ramucirumab dose adjustment, four of the six patients experienced at least one dose delay, but dose reduction or dose omission did not occur. In the case of EGFR-TKIs, one patient experienced two dose reductions for erlotinib, whereas one patient had a dose reduction and one patient a dose interruption for osimertinib. AEs were the most common reason for dose modification.

\subsection{Efficacy}

All patients in the erlotinib plus ramucirumab cohort and two patients in the osimertinib plus ramucirumab cohort had an objective response, resulting in an ORR of 83.3\% (Table 4). All six patients in the study achieved disease control. Figure $1 \mathrm{~A}$ shows the best change in tumor size for all patients with measurable disease at baseline. Tumor response during the treatment period is shown in Figure 1B. With regard to intracranial response, one patient had a measurable untreated CNS lesion associated with brain edema, and this patient showed intracranial tumor shrinkage of $66 \%$ as the best response (PR) accompanied by disappearance of the edema. The other five patients had nonmeasurable lesions, with the CNS response being classified as non-CR/non-PD (progressive disease). At the time of data cutoff, all patients had continued study treatment.

\section{Discussion}

Patients with untreated brain metastases have been excluded from clinical trials featuring antiangiogenic agents because of concerns about potential intracranial hemorrhage. Our phase $1 \mathrm{~b}$ study is the first prospective trial designed to assess the safety of ramucirumab-in particular, with regard to the incidence of CNS hemorrhage-when combined with erlotinib or osimertinib in patients with previously untreated EGFR mutation-positive NSCLC and with asymptomatic brain metastases.

No CNS hemorrhage occurred in the study patients treated with ramucirumab and either erlotinib or osimertinib, suggestive of a minimal or low risk for intracranial hemorrhage associated with 
ramucirumab administration in this patient population. The overall safety profile for both combination therapies was tolerable, consistent with the profiles of each drug in previous trials $[8,15,20,24]$. Our results thus indicate that ramucirumab at a dose of $10 \mathrm{mg} / \mathrm{kg}$ every 2 weeks can be administered safely in combination with erlotinib or osimertinib at full doses in patients with asymptomatic brain metastases. In addition, this study showed that the use of ramucirumab in patients with previously untreated EGFR mutation-positive NSCLC and with brain metastases is acceptable.

The brain is one of the most common sites of distant metastasis in patients with advanced EGFRmutated NSCLC, with such brain metastasis being associated with shorter survival and increased morbidity [25]. Although the standard treatment of radiotherapy is effective for brain metastases, it can give rise to late complications and cognitive decline. In addition, most patients eventually develop new lesions or recurrences of brain tumors after radiotherapy. There is thus an unmet need for development of effective treatments for NSCLC patients with brain metastases.

Osimertinib penetrates the CNS to a greater extent compared with other EGFR-TKIs and shows CNS efficacy as well as systemic efficacy in patients with EGFR mutation-positive NSCLC $[8,10,26]$. However, the median PFS for osimertinib in such individuals with CNS metastases is only 15 months, with treatment eventually resulting in the development of resistance [9]. Preclinical studies have revealed an important role for angiogenesis in the development of CNS metastasis as well as a beneficial effect of antiangiogenic agents on such metastasis in NSCLC [27-29]. Combination treatment with EGFR-TKIs and antiangiogenic agents has shown a greater efficacy compared with EGFR-TKI monotherapy in patients with EGFR-mutated NSCLC $[13,14,20]$. The combination of EGFR-TKIs with bevacizumab has also shown encouraging results in EGFR mutation-positive NSCLC patients with brain metastases [30,31]. On the other hand, patients with brain metastases treated with antiangiogenic agents have been thought to be more susceptible to CNS hemorrhage at sites of intracranial lesions because of the occurrence of a fatal cerebral hemorrhage in a phase 1 study [32]. Previous studies have suggested that an increased risk of intracranial hemorrhage might be associated with the size of metastatic brain tumors in patients with advanced NSCLC [33]. Brain tumors have been found to be more numerous and smaller in NSCLC patients with EGFR mutations than in those with wild-type EGFR [34]. In the present study, regardless of the baseline status of brain metastases, intracranial hemorrhage was not observed in patients treated with ramucirumab in combination with erlotinib or osimertinib not only during the DLT evaluation period but also at 6 months after the initiation of ramucirumab administration. The safety of ramucirumab had not previously been prospectively investigated for patients with brain metastases, even though the addition of ramucirumab has been shown to enhance the efficacy of EGFR-TKIs in clinical trials[20, 24, 35]. The dosage of ramucirumab adopted in the present study differs from that used in the REVEL study, in which it was administered to advanced NSCLC patients at a dose of $10 \mathrm{mg} / \mathrm{kg}$ every 3 weeks in combination with docetaxel in the second-line setting [15]. The safety assessment of our study indicates that administration of ramucirumab at the higher dosage of $10 \mathrm{mg} / \mathrm{kg}$ every 2 weeks in combination with erlotinib or osimertinib is acceptable in patients with untreated EGFR-mutant NSCLC and with asymptomatic brain metastases. Our preliminary findings also hint at the efficacy of ramucirumab for brain metastases. CNS involvement of all study patients was assessed by gadolinium-enhanced MRI, 
with only one individual having a measurable brain lesion of $<20 \mathrm{~mm}$ at baseline brain imaging. This measurable untreated brain lesion was associated with edema and showed shrinkage after treatment for 6 weeks, with the responses of the nontarget brain lesions of the other five patients being classified as non-CR/non-PD. The stability of the brain metastatic lesions of the study patients for at least 6 months suggests a clinically meaningful benefit of study treatment, although these findings will require confirmation with a larger patient population.

Although our results have potentially important implications for ramucirumab administration in patients with brain metastases, the study has several limitations. First, the sample size was small as a result of the study design. The findings should thus be interpreted with caution and require confirmation. However, no previous studies have investigated the safety of ramucirumab in patients with brain lesions. Second, brain metastases of all enrolled patients were small, with a major diameter of $<20 \mathrm{~mm}$, and those of only two patients were untreated before enrollment. The present findings might thus not be generalizable to all patients with EGFR-mutated NSCLC and brain metastases. Finally, the long-term safety of ramucirumab with regard to brain hemorrhage remains to be evaluated definitively.

In summary, we here report the results of a phase $1 \mathrm{~b}$ study investigating the safety and tolerability of ramucirumab in combination with erlotinib or osimertinib for patients with previously untreated EGFR mutation-positive NSCLC that has metastasized to the brain. We found that these combination regimens were feasible in patients with asymptomatic brain metastases including those not previously treated. Our results also suggest that treatment with ramucirumab plus EGFR-TKIs has clinically meaningful efficacy, although further large-scale studies of patients with brain metastases are needed.

\section{Declarations}

\section{Funding:}

This work was supported by Eli Lilly and Company.

\section{Conflict of interest:}

Dr. Kaneda reports grants from Eli Lilly, during the conduct of the study; personal fees from Chugai, Astrazeneca, Novartis Pharma, Bristol-Myers Squibb, Ono, Boehringer Ingelheim, MSD, Taiho, Pfizer, Nippon KAYAKU, and Merck, outside the submitted work. Dr. Sawa reports personal fees from Chugai Pharmaceutical, Daiichi Sankyo, and Nippon Boehringer Ingelheim, all outside the submitted work. Dr. Daga reports personal fees from Chugai, and Ono; grants from Astrazeneca, all outside the submitted work. Dr. Atagi reports grants and personal fees from AstraZeneca, Ono, Taiho Pharmaceutical, Boehringer Ingelheim, Pfizer, Bristol-Myers Squibb, MSD, Eli Lilly, Chugai, and Merck; grants and nonfinancial support from F. Hoffmann-La Roche; personal fees from Hisamitsu, Kyowa Hakko Kirin, Novartis Pharma, and Thermo Fisher Scientific, all outside the submitted work. Dr. Mitsuoka reports personal fees from Ono, Bristol-Myers Squibb, and Kyowa Hakko Kirin; grants from Taiho Pharmaceutical, all outside the submitted work. Dr. Kawaguchi reports grants and personal fees from Chugai Pharma, Taiho 
Pharmaceutical, Boehringer Ingelheim, Ono Pharmaceutical, and Bristol-Myers Squibb Japan; grants, personal fees and non-financial support from Eli Lilly; personal fees from Astra Zeneca, MSD Oncology, Pfizer, Novartis, and Astellas Pharma; grants from Daiichi Sankyo,all outside the submitted work.

All remaining authors have declared no conflicts of interest.

\section{Code availability:}

Not applicable

\section{Ethics approval:}

The study was approved by the institutional review board of each participating institution and was conducted in compliance with the ethical principles of the Declaration of Helsinki, the International Council for Harmonisation Good Clinical Practice Guideline, and applicable local regulations.

\section{Consent to participate:}

Informed consent was obtained from all individual participants included in the study.

\section{Consent for publication:}

All authors critically reviewed and contributed to all drafts, approved the final version, and made the decision to submit the report for publication.

\section{Acknowledgments:}

This study was conducted with support from both the Translational Research Center for Medical Innovation, Foundation for Biomedical Research and Innovation at Kobe, Japan, and the Center for Clinical Research and Innovation, Osaka City University hospital, Osaka, Japan. We thank Chikako Minami and Mieko Nishiu as members of the study support staff.

\section{References}

1. Arbour, K.C. and G.J. Riely, Systemic Therapy for Locally Advanced and Metastatic Non-Small Cell Lung Cancer: A Review. JAMA, 2019. 322(8): p. 764-774.

2. Hsu, W.H., et al., Overview of current systemic management of EGFR-mutant NSCLC. Ann Oncol, 2018. 29(suppl_1): p. i3-i9.

3. Maemondo, M., et al., Gefitinib or chemotherapy for non-small-cell lung cancer with mutated EGFR. N Engl J Med, 2010. 362(25): p. 2380-8.

4. Rosell, R., et al., Erlotinib versus standard chemotherapy as first-line treatment for European patients with advanced EGFR mutation-positive non-small-cell lung cancer (EURTAC): a multicentre, openlabel, randomised phase 3 trial. Lancet Oncol, 2012. 13(3): p. 239-46. 
5. Sequist, L.V., et al., Phase III study of afatinib or cisplatin plus pemetrexed in patients with metastatic lung adenocarcinoma with EGFR mutations. J Clin Oncol, 2013. 31(27): p. 3327-34.

6. Oxnard, G.R., et al., Acquired resistance to EGFR tyrosine kinase inhibitors in EGFR-mutant lung cancer: distinct natural history of patients with tumors harboring the T790M mutation. Clin Cancer Res, 2011. 17(6): p. 1616-22.

7. Yu, H.A., et al., Analysis of tumor specimens at the time of acquired resistance to EGFR-TKI therapy in 155 patients with EGFR-mutant lung cancers. Clin Cancer Res, 2013. 19(8): p. 2240-7.

8. Soria, J.C., et al., Osimertinib in Untreated EGFR-Mutated Advanced Non-Small-Cell Lung Cancer. N Engl J Med, 2018. 378(2): p. 113-125.

9. Reungwetwattana, T., et al., CNS Response to Osimertinib Versus Standard Epidermal Growth Factor Receptor Tyrosine Kinase Inhibitors in Patients With Untreated EGFR-Mutated Advanced Non-SmallCell Lung Cancer. J Clin Oncol, 2018: p. JCO2018783118.

10. Wu, Y.L., et al., CNS Efficacy of Osimertinib in Patients With T790M-Positive Advanced Non-Small-Cell Lung Cancer: Data From a Randomized Phase III Trial (AURA3). J Clin Oncol, 2018. 36(26): p. 27022709.

11. Ichihara, E., et al., Phase II trial of gefitinib in combination with bevacizumab as first-line therapy for advanced non-small cell lung cancer with activating EGFR gene mutations: the Okayama Lung Cancer Study Group Trial 1001. J Thorac Oncol, 2015. 10(3): p. 486-91.

12. Rosell, R., et al., Erlotinib and bevacizumab in patients with advanced non-small-cell lung cancer and activating EGFR mutations (BELIEF): an international, multicentre, single-arm, phase 2 trial. Lancet Respir Med, 2017. 5(5): p. 435-444.

13. Saito, H., et al., Erlotinib plus bevacizumab versus erlotinib alone in patients with EGFR-positive advanced non-squamous non-small-cell lung cancer (NEJ026): interim analysis of an open-label, randomised, multicentre, phase 3 trial. Lancet Oncol, 2019. 20(5): p. 625-635.

14. Seto, T., et al., Erlotinib alone or with bevacizumab as first-line therapy in patients with advanced nonsquamous non-small-cell lung cancer harbouring EGFR mutations (JO25567): an open-label, randomised, multicentre, phase 2 study. Lancet Oncol, 2014. 15(11): p. 1236-44.

15. Garon, E.B., et al., Ramucirumab plus docetaxel versus placebo plus docetaxel for second-line treatment of stage IV non-small-cell lung cancer after disease progression on platinum-based therapy (REVEL): a multicentre, double-blind, randomised phase 3 trial. Lancet, 2014. 384(9944): p. 665-73.

16. Tabernero, J., et al., Ramucirumab versus placebo in combination with second-line FOLFIRI in patients with metastatic colorectal carcinoma that progressed during or after first-line therapy with bevacizumab, oxaliplatin, and a fluoropyrimidine (RAISE): a randomised, double-blind, multicentre, phase 3 study. Lancet Oncol, 2015. 16(5): p. 499-508.

17. Wilke, H., et al., Ramucirumab plus paclitaxel versus placebo plus paclitaxel in patients with previously treated advanced gastric or gastro-oesophageal junction adenocarcinoma (RAINBOW): a double-blind, randomised phase 3 trial. Lancet Oncol, 2014. 15(11): p. 1224-35. 
18. Fuchs, C.S., et al., Ramucirumab monotherapy for previously treated advanced gastric or gastrooesophageal junction adenocarcinoma (REGARD): an international, randomised, multicentre, placebo-controlled, phase 3 trial. Lancet, 2014. 383(9911): p. 31-39.

19. Zhu, A.X., et al., Ramucirumab after sorafenib in patients with advanced hepatocellular carcinoma and increased alpha-fetoprotein concentrations (REACH-2): a randomised, double-blind, placebocontrolled, phase 3 trial. Lancet Oncol, 2019. 20(2): p. 282-296.

20. Nakagawa, K., et al., Ramucirumab plus erlotinib in patients with untreated, EGFR-mutated, advanced non-small-cell lung cancer (RELAY): a randomised, double-blind, placebo-controlled, phase 3 trial. Lancet Oncol, 2019. 20(12): p. 1655-1669.

21. Besse, B., et al., Bevacizumab in Patients with Nonsquamous Non-Small Cell Lung Cancer and Asymptomatic, Untreated Brain Metastases (BRAIN): A Nonrandomized, Phase II Study. Clin Cancer Res, 2015. 21(8): p. 1896-903.

22. Socinski, M.A., et al., Safety of bevacizumab in patients with non-small-cell lung cancer and brain metastases. J Clin Oncol, 2009. 27(31): p. 5255-61.

23. Gubens, M.A., et al., A pooled analysis of advanced nonsquamous non-small cell lung cancer patients with stable treated brain metastases in two phase II trials receiving bevacizumab and pemetrexed as second-line therapy. J Thorac Dis, 2018. 10(1): p. 219-227.

24. Yu, H.A., et al., Phase I Study of the Efficacy and Safety of Ramucirumab in Combination with Osimertinib in Advanced T790M-positive EGFR-mutant Non-small Cell Lung Cancer. Clin Cancer Res, 2021. 27(4): p. 992-1002.

25. Passaro, A., et al., Brain metastases in EGFR-positive non-small cell lung cancer: the way to the sanctuary becomes less winding. Ann Transl Med, 2019. 7(Suppl 3): p. S80.

26. Ahn, M.J., et al., Osimertinib for Patients With Leptomeningeal Metastases Associated With EGFR T790M-Positive Advanced NSCLC: The AURA Leptomeningeal Metastases Analysis. J Thorac Oncol, 2020. 15(4): p. 637-648.

27. Ilhan-Mutlu, A., et al., Bevacizumab Prevents Brain Metastases Formation in Lung Adenocarcinoma. Mol Cancer Ther, 2016. 15(4): p. 702-10.

28. Kienast, Y., et al., Real-time imaging reveals the single steps of brain metastasis formation. Nat Med, 2010. 16(1): p. 116-22.

29. Masuda, C., et al., Bevacizumab suppresses the growth of established non-small-cell lung cancer brain metastases in a hematogenous brain metastasis model. Clin Exp Metastasis, 2020. 37(1): p. 199-207.

30. Chikaishi, Y., et al., Effect of erlotinib plus bevacizumab on brain metastases in patients with nonsmall cell lung cancer. Ann Transl Med, 2018. 6(20): p. 401.

31. Feng, P.H., et al., Bevacizumab Reduces S100A9-Positive MDSCs Linked to Intracranial Control in Patients with EGFR-Mutant Lung Adenocarcinoma. J Thorac Oncol, 2018. 13(7): p. 958-967.

32. Gordon, M.S., et al., Phase I safety and pharmacokinetic study of recombinant human anti-vascular endothelial growth factor in patients with advanced cancer. J Clin Oncol, 2001. 19(3): p. 843-50. 
33. DiLuna, M.L., et al., Prognostic factors for survival after stereotactic radiosurgery vary with the number of cerebral metastases. Cancer, 2007. 109(1): p. 135-45.

34. Sekine, A., et al., Metastatic brain tumors from non-small cell lung cancer with EGFR mutations: distinguishing influence of exon 19 deletion on radiographic features. Lung Cancer, 2012. 77(1): $p$. 64-9.

35. Reck, M., et al., Randomized, Double-Blind Phase Ib/III Study of Erlotinib With Ramucirumab or Placebo in Previously Untreated EGFR-Mutant Metastatic Non-Small-Cell Lung Cancer (RELAY): Phase Ib Results. Clin Lung Cancer, 2018. 19(3): p. 213-220 e4.

\section{Tables}


Table 1.

Patient characteristics at baseline.

\begin{tabular}{|c|c|c|c|}
\hline Characteristic & $\begin{array}{l}\text { Cohort } 1 \\
(\text { ERL+RAM, } n=3)\end{array}$ & $\begin{array}{l}\text { Cohort } 2 \\
(\text { OSM+RAM, } n=3)\end{array}$ & Total $(n=6)$ \\
\hline Median age (range), years & $64(59-77)$ & $63(50-75)$ & $63.5(50-77)$ \\
\hline \multicolumn{4}{|l|}{ Sex } \\
\hline Male & 2 & 2 & 4 \\
\hline \multicolumn{4}{|l|}{ ECOG performance status } \\
\hline 0 & 1 & 2 & 3 \\
\hline 1 & 2 & 1 & 3 \\
\hline \multicolumn{4}{|l|}{ Smoking } \\
\hline Ever & 1 & 1 & 2 \\
\hline Never & 2 & 2 & 4 \\
\hline \multicolumn{4}{|l|}{ Disease classification } \\
\hline Primary & 2 & 2 & 4 \\
\hline Recurrent & 1 & 1 & 2 \\
\hline \multicolumn{4}{|l|}{ EGFR mutation } \\
\hline Exon-19 deletion & 1 & 1 & 2 \\
\hline Exon-21 L858R & 2 & 2 & 4 \\
\hline \multicolumn{4}{|c|}{ Prior treatment of brain metastasis } \\
\hline None & 1 & 1 & 2 \\
\hline Resection & 1 & 0 & 1 \\
\hline Radiotherapy & 1 & 2 & 3 \\
\hline
\end{tabular}

Abbreviations: ERL, erlotinib; RAM, ramucirumab; OSM, osimertinib; ECOG, Eastern Cooperative Oncology Group. 
Table 2.

Safety overview and dose-limiting toxicity (DLT) assessment.

\begin{tabular}{|llll|}
\hline & $\begin{array}{l}\text { Cohort 1 } \\
(\text { ERL+RAM, } n=3)\end{array}$ & $\begin{array}{l}\text { Cohort 2 } \\
(\text { OSM+RAM, } n=3)\end{array}$ & Total $(n=6)$ \\
\hline DLT-evaluable patients & 3 & 3 & 6 \\
\hline Patients with DLT & 0 & 0 & 0 \\
\hline Any TEAE & 3 & 3 & 2 \\
\hline Grade $\geq 3$ TEAE & 1 & 1 & 6 \\
\hline Any TRAE & 3 & 3 & 1 \\
\hline Grade $\geq 3$ TRAE & 1 & 0 & 0 \\
\hline Serious TRAE & 0 & 0 & 0 \\
\hline Discontinued study & 0 & 0 & 2 \\
treatment because of TRAE & & 1 & \\
\hline TRAEs leading to dose & 1 & & \\
adjustment (EGFR-TKI) & & & \\
\hline
\end{tabular}

Abbreviations: ERL, erlotinib; RAM, ramucirumab; OSM, osimertinib; TEAE, treatment-emergent adverse event; TRAE, treatment-related adverse event; EGFR-TKI, epidermal growth factor receptor-tyrosine kinase inhibitor. 
Table 3.

Treatment-emergent adverse events of any grade.

\begin{tabular}{|c|c|c|c|c|c|c|c|c|}
\hline \multirow[b]{3}{*}{ Patients with adverse events } & \multicolumn{3}{|c|}{$\begin{array}{l}\text { Cohort } 1 \\
(\text { ERL+RAM, } n=3)\end{array}$} & \multicolumn{3}{|c|}{$\begin{array}{l}\text { Cohort } 2 \\
(\text { OSM+RAM, n=3) }\end{array}$} & \multirow{2}{*}{\multicolumn{2}{|c|}{$\begin{array}{l}\text { Total }(=6) \\
\text { Any Grade }\end{array}$}} \\
\hline & \multicolumn{2}{|c|}{ Any Grade } & Grade 3 & \multicolumn{2}{|c|}{ Any Grade } & \multirow{2}{*}{$\begin{array}{l}\text { Grade } 3 \\
0\end{array}$} & & \\
\hline & 3 & $(100)$ & 0 & 3 & $(100)$ & & 6 & $(100)$ \\
\hline Rash & 2 & $(66.7)$ & 0 & 3 & $(100)$ & 0 & 5 & $(83.3)$ \\
\hline Dry Skin & 3 & $(100)$ & 0 & 0 & & 0 & 3 & $(50)$ \\
\hline Stomatitis & 3 & $(100)$ & 0 & 0 & & 0 & 3 & $(50)$ \\
\hline Diarrhea & 2 & $(66.7)$ & 0 & 1 & $(33.3)$ & 0 & 3 & $(50)$ \\
\hline Paronychia & 2 & $(66.7)$ & 0 & 1 & $(33.3)$ & 0 & 3 & $(50)$ \\
\hline ALT increased & 2 & $(66.7)$ & 0 & 1 & $(33.3)$ & 0 & 3 & $(50)$ \\
\hline Epistaxis & 2 & $(66.7)$ & 0 & 1 & $(33.3)$ & 0 & 3 & $(50)$ \\
\hline Edema & 1 & (33.3) & 0 & 2 & $(66.7)$ & 0 & 3 & $(50)$ \\
\hline AST increased & 2 & $(66.7)$ & 0 & 0 & & 0 & 2 & (33.3) \\
\hline Pruritis & 2 & $(66.7)$ & 0 & 0 & & 0 & 2 & (33.3) \\
\hline Proteinuria & 1 & (33.3) & 0 & 1 & (33.3) & 0 & 2 & (33.3) \\
\hline Platelet Count Decreased & 1 & (33.3) & 0 & 1 & (33.3) & 0 & 2 & (33.3) \\
\hline Blurred vision & 1 & (33.3) & 0 & 0 & & 0 & 1 & $(16.7)$ \\
\hline Decresed appetite & 1 & (33.3) & 0 & 0 & & 0 & 1 & $(16.7)$ \\
\hline Fatigue & 1 & (33.3) & 0 & 0 & & 0 & 1 & $(16.7)$ \\
\hline Hemorrhoid Bleeding & 1 & (33.3) & 0 & 0 & & 0 & 1 & $(16.7)$ \\
\hline Hypertension & 1 & (33.3) & $1 \quad$ (33.3) & 0 & & 0 & 1 & $(16.7)$ \\
\hline Increased Blood Bilirubin & 1 & (33.3) & 0 & 0 & & 0 & 1 & $(16.7)$ \\
\hline Injection site Bleeding & 1 & (33.3) & 0 & 0 & & 0 & 1 & $(16.7)$ \\
\hline Muscle cramp & 1 & (33.3) & 0 & 0 & & 0 & 1 & $(16.7)$ \\
\hline Alopecia & 0 & & 0 & 1 & $(33.3)$ & 0 & 1 & $(16.7)$ \\
\hline Stomatorrhagia & 0 & & 0 & 1 & $(33.3)$ & 0 & 1 & $(16.7)$ \\
\hline Dizziness & 0 & & 0 & 1 & $(33.3)$ & 0 & 1 & $(16.7)$ \\
\hline Increased Serum Creatinine & 0 & & 0 & 1 & (33.3) & 0 & 1 & $(16.7)$ \\
\hline
\end{tabular}




\begin{tabular}{|lllllllll|} 
Myalgia & 0 & 0 & 1 & $(33.3)$ & 0 & & 1 & $(16.7)$ \\
\hline Arthritis & 0 & 0 & 1 & $(33.3)$ & 0 & & 1 & $(16.7)$ \\
Anaphylaxis & 0 & 0 & 1 & $(33.3)$ & 1 & $(33.3)$ & 1 & $(16.7)$ \\
Headache & 0 & 0 & 1 & $(33.3)$ & 0 & & 1 & $(16.7)$ \\
\hline
\end{tabular}

Abbreviations: ERL, erlotinib; RAM, ramucirumab; OSM, osimertinib; ALT, alanine aminotransferase; AST, aspartate aminotransferase.

Table 4.

Overall and intracranial responses.

\begin{tabular}{|llll|}
\hline Systemic response & $\begin{array}{l}\text { Cohort 1 } \\
(\text { ERL+RAM, } n=3)\end{array}$ & $\begin{array}{l}\text { Cohort 2 } \\
(\text { OSM+RAM, } n=3)\end{array}$ & Total $(n=6)$ \\
\hline Complete response (CR) & 0 & 0 & 0 \\
\hline Partial response (PR) & 3 & 2 & 5 \\
\hline Stable disease (SD) & 0 & 1 & 1 \\
\hline Progressive disease (PD) & 0 & 0 & 0 \\
\hline Overall response & 3 & 2 & 5 \\
\hline Intracranial response & & & 0 \\
\hline Complete response (CR) & 0 & 0 & 1 \\
\hline Partial response (PR) & 1 & 0 & 0 \\
\hline Stable disease (SD) & 0 & 0 & 0 \\
\hline Progressive disease (PD) & 0 & 0 & 5 \\
\hline Non-CR/non-PD & 2 & 3 & \\
\hline
\end{tabular}

Abbreviations: ERL, erlotinib; RAM, ramucirumab; OSM, osimertinib.

\section{Figures}


A

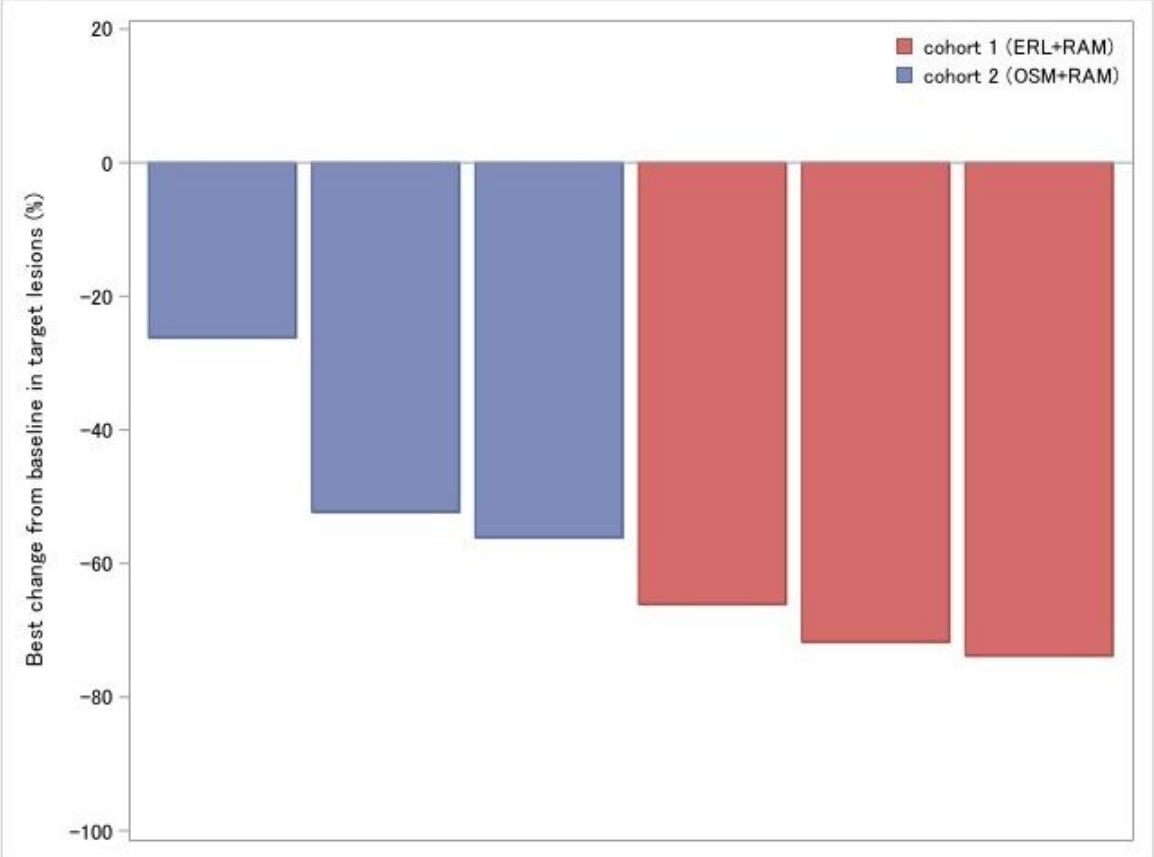

B

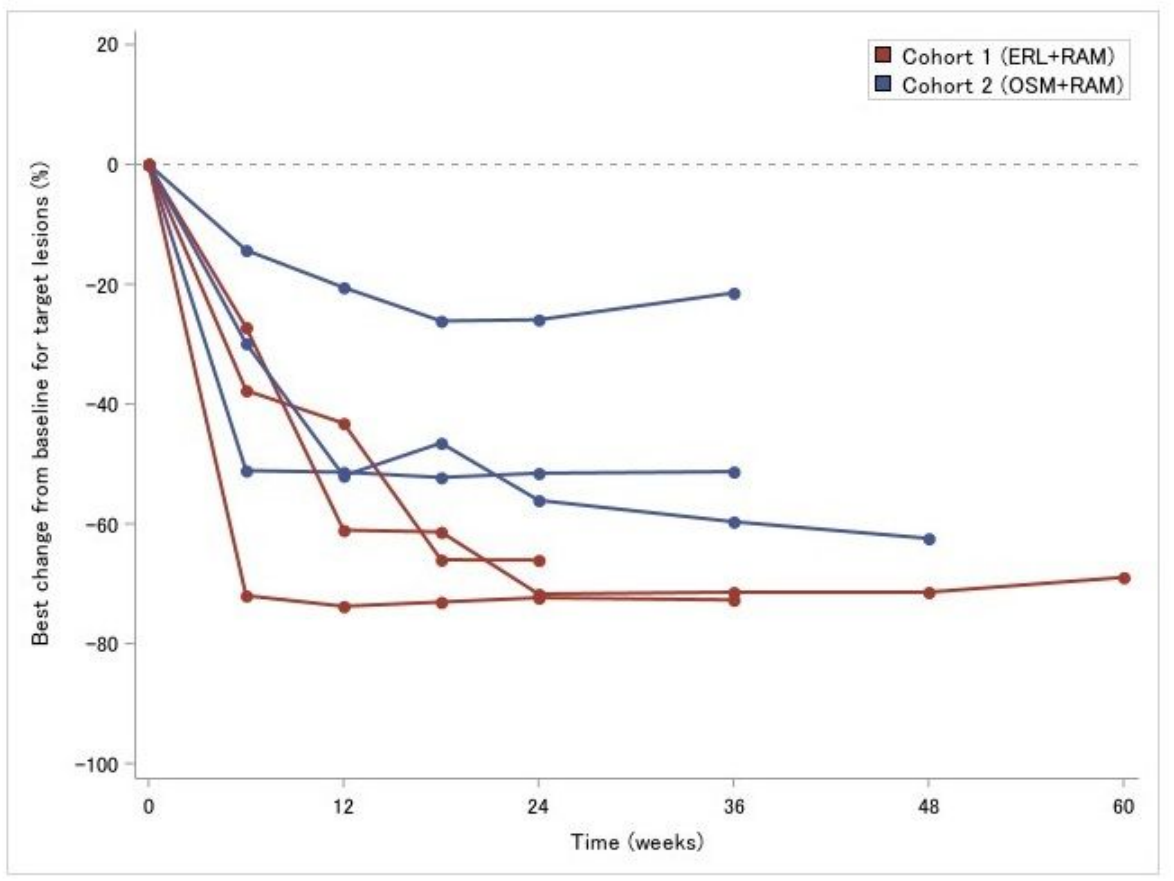

\section{Figure 1}

Best changes in target tumor burden as assessed by investigators. A) Waterfall plot showing best response for target lesions in each cohort. ERL, erlotinib; RAM, ramucirumab; OSM, osimertinib. B) Change in target tumor burden over time in individual patients. 